\title{
Transparenz als Monstranz
}

\section{Warum der lautstarken Debatte über ein Lobbyregister die Zuhörer fernbleiben.}

\author{
Ulrike Propach, Jens Fuderholz
}

\begin{abstract}
Kernaussagen
Die Public-Affairs-Branche, vor allem das Verbändewesen, setzt sich seit langem mit der Forderung nach einem Transparenzregister auseinander. Dieses genießt jedoch selten Priorität. Die hier vorgestellten Ergebnisse der Verbändestudie 2012 zeigen, dass die Verbände im Tagesgeschäft einer Vielzahl anderer Herausforderungen begegnen müssen. Dazu gehören u.a. die sozialen Netzwerke sowie die Schaffung professionellerer Strukturen, die schnelles Handeln im politischen Umfeld in Kombination mit der PR verbinden. Vor allem aber geht es ihnen um die Kommunikation mit ihren Mitgliedern, der Politik und Multiplikatoren. Die Autoren plädieren dafür, die Debatte um ein Lobbyregister in die Diskussionen um eine sich wandelnde politische Kultur in Deutschland einzubetten.
\end{abstract}

\section{Einführung}

Die Public-Affairs-Branche, vor allem das Verbändewesen, hat sich in den vergangenen Jahren in Diskussionen immer wieder mit der Forderung nach einem Transparenzregister auseinandergesetzt. Wenn man die Ergebnisse der gesamten Verbändestudie 2012 ansieht, kämpfen aber die Verbände in ihrem Tagesgeschäft an vielen anderen Fronten: die Herausforderung der Omnipotenz sozialer Netzwerke (an denen jedoch nur ein Viertel der Verbände teilnimmt) oder die Schaffung von professionelleren Strukturen, die schnelles Handeln im politischen Umfeld in Kombination mit der PR verbinden. Vor allem aber geht es ihnen um die Kommunikation zu ihren Mitgliedern, der Politik und Multiplikatoren. Dass dabei die manchmal zu akademisch geführte Diskussion um ein Lobbyregister ins Hintertreffen kommt, ist nachvollziehbar. Die Autoren plädieren dafür, die Debatte nicht nur darauf zu beschränken, sondern insgesamt die politische Kultur in Deutschland anzusehen.

\section{Wechsel des Politikstils}

Die 16. Bundestagswahlperiode hat einen Wandel des Politikstils mit sich gebracht. Von vielen Medien und Lobbyisten vor der Wahl 2009 noch mehr oder weniger vorsichtig als ,wirtschaftsfreundlich“ tituliert, wurde die Koalition von CDU/ CSU und FDP danach als lobbygesteuerte Regierung eingestuft, die vor allem Klientelpolitik betreibe. Nach der Causa „Mövenpick“ (auf eine große Wahlkampfspende folgte die Reduzierung der Mehrwertsteuer auf Übernachtungen von
$19 \%$ auf $7 \%$ ) blieb dieses Etikett - ob nun real oder von der Opposition zugeschrieben - an der Regierung hängen.

Der schnell aufgestiegene und schon wieder am politischen Himmel verglühte Stern Karl-Theodor zu Guttenberg zeigt wie kein zweiter, welchen Versuchungen die heutige politische Klasse ausgeliefert ist: In einer unglaublich schnellen Medienkultur, die durch Facebook und Twitter nochmals an Tempo gewonnen hat, ringen alle um Aufmerksamkeit. Dass in einem eineinhalbminütigen Nachrichtenbeitrag politische Inhalte zumeist nicht in ihrer tatsächlichen Komplexität abzubilden sind, ist eigentlich jedem klar. Und dennoch leben wir in diesem Mediensystem und erliegen ihm auch manchmal.

Dass die Bürger sich nach leuchtenden Figuren sehnen, die ihnen in dieser politikverdrossenen Zeit das Gegenteil beweisen, ist ebenfalls ersichtlich. Die Ernüchterung und der Zorn sind jedoch groß. Die „Causa Wulff“ konnte möglicherweise erst in der Posttraumatisierung der „Guttenplag“-Ära so bedeutend werden. Und sie ist wiederum ein Zeichen dafür, dass die Bürger genug haben von Politikern, die nur noch einem System aus Wirtschaft und Politik und nicht mehr ihnen - als dem eigentlichen Souverän - dienen. Vor dieser Herausforderung stehen nun auch Verbände, die die verschiedenen Interessen der Bevölkerung sowie der Wirtschaft repräsentieren wollen. Sie bündeln und artikulieren diese gegenüber Politik und Verwaltung. Besonders wichtig für diese Rolle ist ihre Sonderstellung in der deutschen Demokratie. Die Geschäftsordnung des Deutschen Bundestags sieht ihre Existenz ausdrücklich vor und weist den Verbänden eine herausgehobene Stellung zu. Sie sind sozusagen die „ordnungsgemäßen“ Lobbyorganisationen - durchaus im Gegensatz zu Kanzleien, Beratern oder sogar NGOs. Die Frage, wie diese Verbände einer Kontrolle von Lobbying gegenüberstehen, kann also ein wertvoller Beitrag zur Debatte sein.

\section{Ergebnisse der Verbändestudie 2012 zum Lobbyregister}

Die Debatte um ein Lobbyregister in Deutschland ist nicht leiser geworden. In den vergangenen Jahren hat sie sich eher zugespitzt. Dies hat mehrere Ursachen. Zum einen hat das freiwillige Register der EU, das seit 2008 zuerst nur für die Kommission galt, durch die Zusammenführung mit dem Parlament im Juni 2011 und den verschärften Vorschriften für Lobbyisten Veränderungen gebracht. ${ }^{1}$ Zum anderen hat die

1 Siehe hierzu die Website des Lobbyregisters http://europa.eu/transparency-register/index_de.htm sowie die zugehörige Pressemitteilung der Europäischen Kommission (http://europa.eu/rapid/pressReleasesAction.do? reference $=I P / 11 / 773 \&$ format $=H T M L \&$ aged $=1 \&$ language $=$ DE\&guilanguage $=e n)$. 
Opposition im Deutschen Bundestag durch eigene Anträge für die Aufrechterhaltung der Diskussion gesorgt. Auch hat sich die Branche im Rahmen der de'ge'pol (Deutsche Gesellschaft für Politikberatung) und des DPRG-Arbeitskreises Public Affairs sowie angeregt durch Initiativen von außen von transparency international und LobbyControl weiter stark mit dem Thema beschäftigt. Statt einer Vereinheitlichung der Meinung haben sich die Positionen dabei weiter voneinander entfernt. Dies lässt sich ablesen aus der Gegenüberstellung der Daten aus der Verbändestudie 2009 und der Erhebung aus dem Spätsommer 2011, die in der Verbändestudie 2012 publiziert wurden (vgl. Fuderholz \& Propach, 2009; Fuderholz \& Propach, 2012). ${ }^{2}$

\section{Abbildung 1: Wie finden Sie die Pläne für ein Lobbyregister in Deutsch- land?}

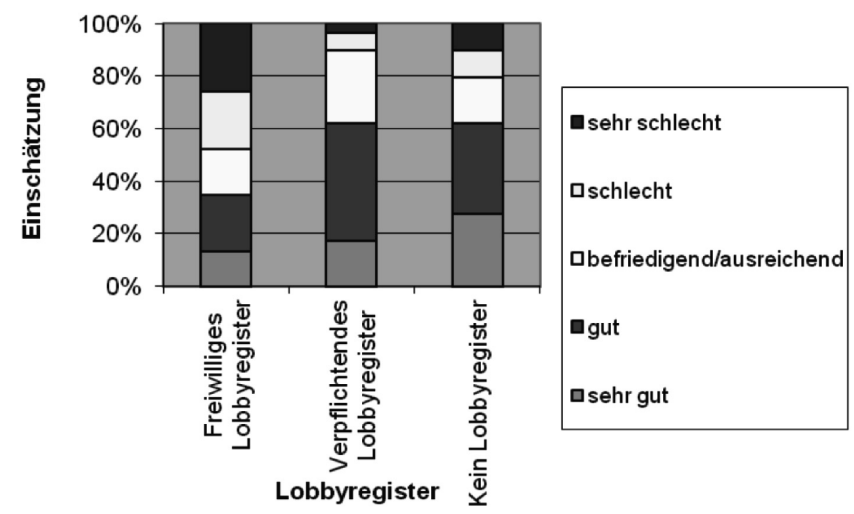

Das freiwillige Lobbyregister wird wesentlich schlechter bewertet als ein verpflichtendes oder gar keines. Interessanterweise sind die Werte der Befürwortung für kein oder ein verpflichtendes Lobbyregister gleich groß. Einigkeit in der Branche sieht anders aus. Im Vergleich zu den Werten aus 2009 ist die Zustimmung zu einem verpflichtenden Lobbyregister gleich geblieben, jedoch ist die Befürwortung eines freiwilligen Registers um 25\% zurückgegangen. Die Vermeidung eines Lobbyregisters wurde 2009 mit 10\% (sehr gut, gut) eingeschätzt, in 2012 sind es 41 Prozentpunkte mehr!

Abbildung 2: Welche Meinung haben Sie zum bereits bestehenden Lobbyregister der EU?

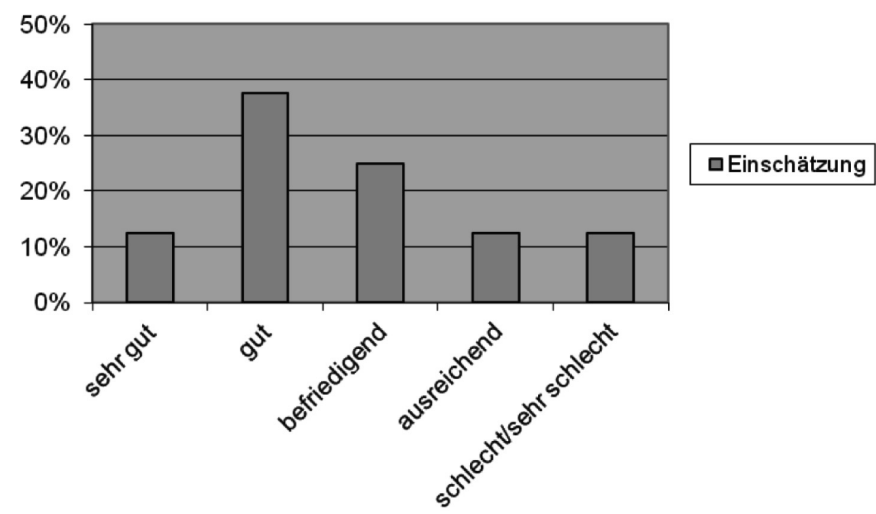

Die Wertschätzung des EU-Lobbyregisters hat sich gegenüber 2009 verändert - insgesamt wird das EU-Register mit 50\% Zustimmung (gut, sehr gut) wesentlich freundlicher bewertet als noch in 2009 mit 35\% (gut, sehr gut). Die negative Einschätzung ist jedoch von $20 \%$ in 2009 auf $25 \%$ in 2011 leicht gestiegen.

$\mathrm{Ob}$ das EU-Register nun wirklich als Erfolg gefeiert werden kann, sei dahin gestellt. Mit wenigen hundert Eintragungen gestartet, wurde bei der Pressekonferenz am 23. Juni 2011 zur Zusammenführung des freiwilligen Registers der EUKommission mit dem neuen für das EU-Parlament die Teilnehmerzahl mit 4.000 beziffert. Im März 2012 hatte das Register 4.560 Eintragungen. Gespiegelt an den nationalen Zahlen von den 27 EU-Mitgliedern der Verbände, NichtRegierungsorganisationen sowie Unternehmen, die in Brüssel und Strasbourg Einfluss nehmen, ist diese Zahl jedoch ernüchternd gering.

Die seit Jahren nicht abebbende Diskussion um die Schaffung eines deutschen Lobbyregisters ist ein interessantes Phänomen. Im Expertengespräch der Verbändestudie 2012, das im Juli 2011 in Berlin durchgeführt wurde, gab es zu Beginn eher verhaltene Reaktionen auf das Thema. Jedoch wurde dann in einem intensiven Diskurs herausgearbeitet, dass die Branche (Verbände genauso wie darin beteiligte Unternehmen) sich sehr wohl dazu äußern müssten. Stefan Reker, Pressesprecher des Verbandes Privater Krankenkassen und langjähriger politischer Journalist stellt fest: „Hinter Forderungen nach Registern und Regulierungen für die Lobby steckt leider oft nicht nur der berechtigte Wunsch nach Transparenz, sondern eine pauschale Verdächtigungs-Attitüde, bei manchen sogar ein Versuch der Kriminalisierung. Das mündet dann in die Illusion, man müsse nur allerlei regulieren und verbieten, um Missbräuche zu unterbinden.“ Claudia Konrad-Kreml, Geschäftsführerin einer international arbeitenden Agentur, führt einen weiteren Aspekt ins Feld: „Inwieweit es ausschließlich um die Legitimierung von Interessensvertretung geht, muss mit einem Fragezeichen versehen werden." Die Vermischung von Forderungen von LobbyControl und Transparency International nach der tatsächlichen Notwendigkeit von Transparenz politischer Entscheidungsprozesse und „Transparenz als Monstranz“ im Sinne gesellschaftlicher In-Begriffe ist ernsthaft in Frage zu stellen.
2 Der besseren Übersicht ist geschuldet, dass im weiteren Verlauf immer von der Verbändestudie 2012 und gleichlautend von den Daten „2012“ gesprochen wird, obwohl der Zeitpunkt der Datenerhebung im Jahr 2011 liegt. 


\section{Auf einen Blick: Die Kernaussagen der Verbändestudie 2012}

Mitgliederwerbung und-kommunikation werden wichtiger

Die wichtigsten Adressaten sind immer noch Mitglieder, Politik und Medien - in dieser Reihenfolge. Vereinsmitglieder werden jedoch weniger persönlich angesprochen: Der Newsletter verdrängt nationale und regionale Events. Social Media spielen ebenfalls eine eher geringe Rolle in der internen Verbandskommunikation. Die am weitesten verbreiteten Instrumente sind E-Mail für die kurzfristige Information und Mitgliederversammlungen als wesentlicher Bestandteil der innerverbandlichen Willensbildung.

\section{Politische Kommunikation wird erfolgreicher}

Dies bestätigt die Umfrage: 6o Prozent der Befragten, die an einen Zusammenhang zwischen politischen Entscheidungen und medialer Präsenz glauben, schätzen den Erfolg ihrer politischen Kommunikation als gut oder sehr gut ein. Hierbei ist interessant, dass sowohl Funktionäre als auch operative Geschäftsführer für politische Kommunikation zuständig sind. Offenbar versuchen Verbände durch eine stärkere Einbindung der strategischen Ebene ihre Meinung im wachsenden Wettbewerb durchzusetzen. Die Einschätzung des Erfolgs auf Bundes-, Länder- und kommunaler Ebene ist dahingegen leicht gesunken. Recht deutlich ist die Einschätzung des Erfolgs auf europäischer Ebene zurückgegangen. Der Anteil derer, die ihre politische Kommunikation auf der EU-Ebene mindestens als „erfolgreich“erachten, ist um rund zehn Prozentpunkte gesunken. Gerade in der höchsten Kategorie wird dies sichtbar: Vor drei Jahren bewerteten rund 15 Prozent ihre Kommunikation als „sehr erfolgreich“, heute sind es dagegen nicht einmal mehr zehn Prozent.

\section{Bedeutungsgewinn und Professionalisierung der PR}

Public Relations haben gegenüber 2009 deutlich an Bedeutung gewonnen und sind professioneller geworden. Öffentlichkeitsarbeit wird 2012 sogar höher als Mitgliederbindung oder Mitgliedergewinnung eingeschätzt. Presse- und Öffentlichkeitsarbeit sind dabei immer auf den Systemerhalt ausgerichtet: Einfluss und Imagesteigerung sind vorrangige Ziele. Zuständig für Öffentlichkeitsarbeit ist heute nicht mehr nur der Geschäftsführer. Viele Verbände beschäftigen hier Pressesprecher. Doch auch die Auslagerung auf externe Agenturen hat stark zugenommen. Die Verbände haben erkannt, dass ihre Pressearbeit am effektivsten ist, wenn sie ihre Inhalte ansprechend aufbereiten und einen festen Ansprechpartner haben, der in einem ständigen Kontakt mit den Medien steht

\section{Lobbystrategien wachsen in ihrer Bedeutung}

So setzten beispielsweise 9o,g Prozent der Befragten, die politische Kommunikation initiativ starten, auf eine proaktive Lobby-Strategie. Gleichzeitig wird Lobbyarbeit immer mehr versachlicht und Lobbystrategien werden immer weniger dazu verwendet, Protest oder positive Botschaften zu vermitteln. Bei passiven Lobbystrategien sind diese Ziele gar nicht vorhanden. Verbände bedienen sich eher einer sachlichen und rationalen Argumentation, anstatt an die Emotionen der Menschen zu appellieren. So konnten Gesetzesvorschläge und „Was-wäre-wenn"-Szenarien als Kommunikationsmittel bei proaktiven Lobbystrategien deutlich zulegen.

Die Kanäle der Medienarbeit haben sich deutlich verändert

Das Fax und der klassische Postbrief haben als Kommunikationskanal ausgedient. Das Presseportal gewinnt in den letzten drei Jahren merklich an Bedeutung. So ist es nach der E-Mail der wichtigste Kanal für Pressearbeit. Interessanterweise haben sich jedoch trotz starker Veränderung der Kom munikationskanäle die Adressaten kaum verändert: Fachpresse, die (digitale) Öffentlichkeit, überregionale Tageszeitungen und Nachrichtenagenturen sind weiterhin die wichtigsten Adressaten.

Monitoring hat sich verändert und an Bedeutung gewonnen

Um die Übersicht über die Entwicklung relevanter Themen in den Medien zu behalten, greifen mehr Verbände auf Monitoring zurück. Hierbei hat sich der Eigenrecherche-Anteil gegenüber 2009 deutlich reduziert, ist aber weiterhin die verbreitetste Art des Monitoring. Um die Hälfte gesunken ist der Anteil derjenigen, die einen professionellen Dienstleister für Online-Monitoring beauftragen. Bemerkenswert ist, dass immerhin 48,6 Prozent der Befragten tagesaktuelles Monitoring betreiben. Lediglich ein Drittel betreibt jedoch Erfolgskontrolle und verfügt über überprüfbare Ziele.

\section{Wissen um Gesetzgebungsprozesse lässt nach}

Nicht besonders gut steht es um das Wissen um Gesetzgebungsprozesse. Vor allem der Kenntnisstand über die Gesetzgebungsverfahren auf Europaund Bundesebene hat nachgelassen. Die niedrigen Werte zu Kenntnissen über die Gesetzgebung auf Europaebene sind erstaunlich, bedenkt man dass rund 70 Prozent der Gesetze, die Deutschland betreffen, in der EU auf den Weg gebracht werden. Lediglich auf der Länderebene ist ein leichter Aufwärtstrend zu beobachten. Ungefähr gleich geblieben ist der Kenntnisstand der Prozesse in öffentlichen Verwaltungen, Ministerien und politischen Organisationen/Parteien.
Die Konkurrenz unter den Verbänden nimmt zu

Die Zahl der Verbände, die mehr als drei Konkurrenzverbände angeben, ist um mehr als zehn Prozent gestiegen. Vor allem interessant sind die Auswirkungen, die sich daraus ergeben. Je mehr Konkurrenz der Verband besitzt, desto professioneller ist seine Kommunikationsarbeit. Verbände die im Wettbewerb mit anderen stehen, besitzen auch eine professionellere Herangehensweise. So betreiben 61 Prozent der Verbände, die mindestens einen Konkurrenzverband haben, Issues Management. Umgekehrt betreiben 73 Prozent der Verbände, die keine Konkurrenz haben, auch kein Issues Management.

Social Media sind spannend, werden aber kaum genutzt

Das Thema Social Media hat im Expertengespräch, das im Rahmen der Verbändestudie stattfand, eine intensive Diskussion ausgelöst. Allein diese Reaktion zeigt, welchen Wahrnehmungszuwachs das Thema Social Media zu verbuchen hat. Diese Tatsache und steigende Mitgliederzahlen von Online-Communites lassen Social Media zunehmend attraktiver für die Verbandskommunikation erscheinen. Derzeit sind es allerdings eher die NGOs, die neue Medien für ihre Kampagnenführung nutzen. So besitzt nicht einmal ein Viertel aller Befragten einen Verbandsaccount, gut die Hälfte besitzt hingegen einen privaten Account. 14 Prozent führen derzeit schon Kampagnen in Social Media durch, fast 44 Prozent planen dies kurz-, mittel- oder langfristig. Genauso hoch ist auch der Anteil derjenigen, die keine Kampagnen in Social Media planen. Bei der Beobachtung von Social Media wird überwiegend auf freie Dienste zugegriffen. Auffallend ist jedoch, dass 20 Prozent Social Media in ihr Monitoring überhaupt nicht mit einbeziehen.

\section{Lobbying als Politikstil}

Die in der so genannten „Lobby-Studie“ vom Autorentrio Kolbe, Hönigsberger und Osterberg vorgelegten Eckpunkte einer gesamten Transparenz-Regelung bieten viel Zündstoff für eine engagierte Diskussion (Kolbe, Hönigsberger \& Osterberg, 2011). Sie erstellten in Finanzierung der Otto Brenner Stiftung eine „Marktordnung für Lobbyisten - wie Politik den Lobbyeinfluss regulieren kann“. Die Literaturliste verweist auf viele einseitige Quellen, die Sammlung der Eckpunkte lehnt sich in wesentlichen Punkten an die Forderungen der de'ge'pol an. Diese Gesellschaft hat weniger als 150 Mitglieder, vor allem aus Berlin. Gemessen an den schätzungsweise 40.000 im deutschen Public-Affairs-Geschäft tätigen Personen (Mitarbeiter von Verbänden, Unternehmen, Stiftungen, Agenturen sowie Berater; wissenschaftliche Gutachter sind hier ausgenommen) ist dies eine kleine Interessensgruppe. Im Vergleich dazu hat die Deutsche Public Relations Gesellschaft mit etwa 4.000 Mitgliedern das wesentlich größere Gewicht.

Was in der Lobby-Studie nicht zum Tragen kommt, ist, ob solche Regelungen tatsächlich den Problemen begegnet, die sich heute mit Politikstilen und -vermittlung verbinden. Denn die Frage, warum die Politikprozesse von Bürgern nicht mehr verstanden und somit auch nicht mehr mitgetragen werden, wird von vorneherein nicht thematisiert. Die Komplexität von Public Affairs mit einem Register zu vereinfachen, wird nicht gelingen. In einem Zeitalter, wo Politik in Berlin zumeist nur umgesetzt wird, weil Richtlinien der EU umgesetzt werden müssen, setzen Regelungen im Bundestag an der falschen Stelle an. Nationale Themen sind zumeist der globalisierten Welt geschuldet: Verteidigungspolitik, Menschenrechte, Migration, Finanzkrise, Euro-Krise, Entwicklungspolitik, Naturund Umweltschutz, Energiewende u.a. Die Themenliste ließe sich beliebig erweitern. Wer meint, dass wir in nationaler Nabelschau unsere großen Politikfragen lösen können, irrt.

Stefan Reker befürchtet in unserem Expertengespräch durch starke Regulierungen im Lobbyismus einen Kulturver- 
lust: „Solche Regulierungswut würde den Lobbyismus nicht verhindern - nicht in seinen sauberen und schon gar nicht in seinen schmutzigen Formen. Am Ende wäre es wie in den finsteren Zeiten der Prohibition in den USA: Man würde dann eben nicht mehr in den normalen Berliner Büros oder Restaurants miteinander reden, sondern in irgendwelchen Kellergewölben. "Verminderter Zugang zu Abgeordneten, Ministerien und Behörden führe nicht zu einer Verbesserung und erhöhter Durchlässigkeit, sondern bewirke eine stärkere Geheimkultur und noch geringeren Austausch von Bürokratie mit gesellschaftlichen Gruppierungen und der Wirtschaft.

Erstaunlich ist nach wie vor, dass in der Debatte beharrlich ignoriert wird, dass PR-Schaffende bereits seit Jahrzehnten unter Einhaltung von Ehrenkodizes arbeiten. Viele sind entweder in Berufsverbänden, die den Code d'Athene bzw. Code de Lisbonne als Grundlage ihrer Arbeit anerkennen, organisiert oder in Journalistenverbänden akkreditiert. Dort gelten ebenfalls klare Regeln. Derzeit werden diese Regelungen zusammengefasst in einem Kommunikationskodex, zu dem die Konsultationen laufen. Große Unternehmen haben bereits einen „Code of Conduct“ oder Compliance-Regelungen, die klar das Geschäftsgebaren vorgeben. Vertreter von Behörden haben ebenfalls klare Vorgaben, welche Form von Geschenken oder welche Höhe von Einladungen sie annehmen dürfen.

Ebenfalls wird nicht diskutiert, ob die so genannten „Freiheitsrechte" nicht die gleiche Forderungshöhe rechtfertigen, wie die angeblich moralisch unfehlbaren „Transparenzrechte“. Die Vertrauensfähigkeit und damit gelebte Vertraulichkeit, die Grundlage menschlicher Beziehungen ist, gehört zum politischen Geschäft. Wenn nun jeder für politische Zwecke ausgegebene Euro offen gelegt werden soll, stellt sich die Frage, wer daraus Schlüsse ziehen kann: der gemeine Bürger? Oder wiederum Interessen vertretende Organisationen, die sich über derlei Informationen freuen? Die Lobbystudienautoren Kolbe, Hönigsberger und Osterberg verzichteten auf diese Forderung, die durchaus noch von anderen Seiten vertreten wird. Wenn diese aber keine „Footprints“ in Gesetzen haben möchten, um die Herkunft von Ideen nachzuvollziehen, wie soll dann Transparenz hergestellt werden?

\section{Fazit}

Eigentlich müssten wir keine Debatte um Transparenzregister und das Verbot von „Leihbeamten“ führen. Stattdessen ist es dringend geboten, dass sich die politische Klasse sowie die Kommunikatoren zuallererst über die Vermittlung von politischen Inhalten und Strukturen auseinandersetzen und die Bürger wieder neu für Politik begeistern. Denn Politik will nichts anderes als sich mit den Fragen auseinander zu setzen, wie wir in unserer Gesellschaft miteinander leben wollen. In all den Interview-Schlachten und Talkshows haben wir im letzten Jahrzehnt einiges an politischer Kultur verloren - und vor allem den Kontakt zum Volk, für das die gewählten Vertreter eigentlich ihre Arbeit tun.

Dass die Auseinandersetzung über ein Lobbyregister in einem akademisch angehauchten und von den (Anti-)Lobby-
Lobbyisten getriebenen Diskurs stattfindet, zeigen die Ergebnisse der Verbändestudie 2012 übrigens auch sehr deutlich: Die Debatte ist nicht leiser geworden, es hören nur immer weniger Menschen hin. Das Lobbyregister ist nicht oben auf der Agenda der Akteure, sondern eher eine Randbedingung des eigenen Schaffens, die wachsam beobachtet, nicht jedoch zuvorderst aktiv gestaltet werden muss. Viel wichtiger ist den „ordnungsgemäßen“ Verbands-Lobbyisten, wie Inhalte und Themen erfolgreich platziert werden können und wie dafür um Zustimmung geworben werden kann.

Noch eine Erkenntnis bleibt: Der Bürger - als Souverän auch in der Mediendemokratie - findet immer einen Weg, Transparenz herzustellen: ob plagiierende Politiker oder Klientel-Politik, ob die direkte „Bürgerbewegung“ im Internet oder der medial inszenierte Aufschrei. Die Wahrheit findet immer ihren Weg ans Licht. Kann ein Lobbyregister dazu wirklich einen hilfreichen Beitrag leisten?

\section{Literatur}

Fuderholz, J \& U. Propach (2009). Verbändestudie 2009. Fürth: Hirschenverlag.

Fuderholz, J. \& U. Propach (2012). Verbändestudie 2012. Fürth: Hirschenverlag.

Kolbe, A.; H. Hönigsberger \& S. Osterberg (2011). Marktordnung für Lobbyisten. Otto Brenner Stiftung. http://www.otto-brenner-shop.de/ uploads/tx mplightshop/AH 70_Lobby_End.pdf (zuletzt abgerufen am 14.4.2012)

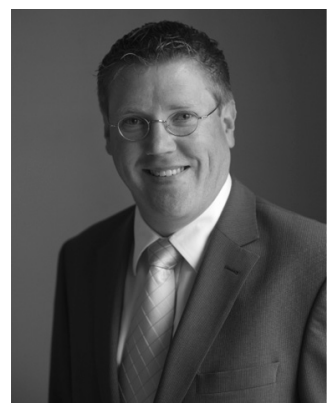

Jens Fuderholz ist Diplom-Soziologe und Gründer, Gesellschafter und Geschäftsführer der TBN Public Relations $\mathrm{GmbH}$, spezialisiert auf Bto-B-Kommunikation. Seit 2002 hat er einen Lehrauftrag für Kommunikationswissenschaft an der OttoFriedrich-Universität Bamberg. Seit 2012 ist er zusätzlich Geschäftsführer der TBN Public Affairs $\mathrm{GmbH}$ \& Co.KG. Email: fuderholz@tbnpa.de

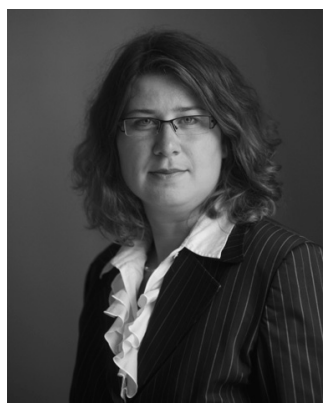

Ulrike Propach ist Soziologin und Geschäftsführerin der TBN Public Affairs GmbH \& Co. KG. Bei der EXPO 2000 arbeitete sie von 1999 bis $2000 \mathrm{im}$ Protokoll der Gesellschaft für die Weltausstellung. Von 2003 bis 2004 leitete sie das Büro der Bundestagspräsidentin a.D., Prof. Dr. Rita Süssmuth. Seit 2001 ist sie als PR- und Politikberaterin und freie Journalistin tätig und hat einen Lehrauftrag für Pu blic Affairs an der Otto-Friedrich-Universität Bamberg. Email: propach@tbnpa.de 